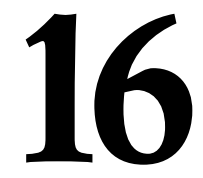

\title{
EL PROGRAMA AUDIT DE LA ANECA Y LAS NORMAS INTERNACIONALES ISO 9000. ANÁLISIS COMPARATIVO
}

\author{
(THE AUDIT PROGRAM OF ANECA AND THE INTERNATIONAL STANDARDS \\ ISO 9000. A COMPARATIVE ANALYSIS)
}

Jorge Antonio Arribas Díaz

Fundación Escuelas Profesionales Sagrada Familia (SAFA), Écija, Sevilla

Catalina Martínez Mediano

Universidad Nacional de Educación a Distancia (UNED)

DOI: 10.5944/educXX1.14609

Cómo referenciar este artículo/How to reference this article:

Arribas Díaz, J. A. y Martínez Mediano, C. (2015). El programa AUDIT de la ANECA y las normas internacionales ISO 9000. Análisis comparativo. Educación XX1, 18(2), 375-395, doi: 10.5944/ educXX1.14609

Arribas Díaz, J. A. y Martínez Mediano, C. (in press). El programa AUDIT de la ANECA y las normas internacionales ISO 9000. Análisis comparativo [The audit program of ANECA and the international standards ISO 9000]. A comparative analysis. Educación XX1, 18(2), 375-395, doi: 10.5944/educXX1.14609

\section{RESUMEN}

Los autores realizan un análisis comparativo entre el programa AUDIT de la Agencia Nacional de Evaluación de la Calidad y Acreditación (ANECA) y la familia de Normas ISO 9000. Tanto AUDIT como ISO sirven de base para la elaboración, implantación, desarrollo y mejora de sistemas de gestión o garantía de la calidad, en este caso en el sector universitario nacional. Así, el presente trabajo, tras describir el programa AUDIT y las normas ISO, se centra en la comparación de los principios, objetivos, requisitos, fases y documentos de ambos acercamientos a la calidad total. Tras la comparación surgen algunas reflexiones sobre los aspectos positivos de AUDIT.

\section{PALABRAS CLAVE}

Calidad educativa; educación superior; gestión de la calidad total; aseguramiento de la calidad; certificación; sistemas de gestión. 


\section{ABSTRACT}

The authors make a comparative analysis between AUDIT program of the National Agency for Quality Assessment and Accreditation (ANECA), and the family of standards ISO 9000. Both AUDIT and ISO serve as a basis for the production, implementation, development and improvement of quality management or assurance systems, in this case at the national university sector. Thus, this present paper, after briefly describing the AUDIT program and ISO standards, focuses on the comparison of the principles, objectives, requirements, phases and documentation of both approaches to total quality. After the comparison some reflections arise on the strengths of AUDIT.

\section{KEY WORDS}

Educational quality; higher education; total quality management; quality assurance; certification; management systems.

\section{INTRODUCCIÓN}

En la Declaración de Bolonia (1999), los Ministros Europeos de Educación se comprometieron a desarrollar para 2010 un Espacio Europeo de Educación Superior común (EEES). Este EEES ha supuesto la transformación de todo el sistema de educación superior, desde los títulos para hacerlos comparables, en grados, másteres y doctorado, a una formación basada en competencias, atendiendo a las necesidades de los empleadores en la sociedad del conocimiento. También ha supuesto el reforzamiento de la movilidad de estudiantes y del profesorado, y la cooperación europea para garantizar la calidad de los diseños, procesos y resultados de la educación superior mediante el desarrollo de criterios y metodologías comparables.

En la reunión de Berlín (2003) de ministros europeos signatarios de Bolonia, la European Network for Quality Assurance in Higher Education (ENQA) fue invitada, en cooperación con la European University Association (EUA), la European Association of Institutions in Higher Education (EURASHE), y la European Students' Union (ESU), para que desarrollase "un conjunto consensuado de criterios, procedimientos y directrices para la garantía de calidad» $\mathrm{y}$ "a explorar los medios que garanticen un sistema adecuado de revisión por pares de garantía de calidad y/o para las agencias u organismos de acreditación», teniendo en cuenta la experiencia de otras asociaciones y redes de garantía de calidad (ENQA, 2005, p. 3).

«La garantía de calidad en la educación superior en absoluto es solo una preocupación europea. En todo el mundo se manifiesta un creciente interés en la calidad... si Europa desea lograr su aspiración de convertirse en la eco- 
nomía basada en el conocimiento más dinámica del mundo (Estrategia de Lisboa), la educación superior europea necesita demostrar que toma en serio la calidad de sus programas y títulos y que se compromete a poner en marcha los medios que aseguren y demuestren esa calidad» (ENQA, 2005, p. 9).

Por otro lado, la evaluación de la calidad de las universidades españolas, a través de sus titulaciones, viene realizándose mediante el Plan Nacional de Evaluación de la Calidad de las Universidades desde 1996, con sucesivas mejoras en subsiguientes planes dentro del Programa de Evaluación Institucional de la ANECA (Agencia Nacional de Evaluación de la Calidad y Acreditación).

Estos Planes de Evaluación de Calidad tienen sus fundamentos en la disciplina académica de la Evaluación de Programas. De la veintena de modelos de evaluación de programas desarrollados en esta disciplina, destacamos uno de los más completos, el enfoque de evaluación de programas orientado a la toma de decisiones para la mejora de los programas y su gestión, representado por Stufflebeam et al. (1971) y Alkin (1969).

Ambos autores destacan los aspectos claves de la organización de los centros y la toma de decisiones sobre los cambios y mejoras de los programas educativos tras su evaluación. En el objetivo principal de este enfoque de evaluación orientada a la decisión, subyace el interés por maximizar la utilización de los resultados de la evaluación, más allá de la mera elaboración y presentación del informe sobre los resultados, pretendiendo que el evaluador oriente a llevar a la práctica las mejoras que deben hacerse en las organizaciones y en los programas, a partir de los resultados de la evaluación, de modo que el evaluador colabore con la dirección del centro y aquellos que deben tomar decisiones de cambio para la mejora en las organizaciones educativas.

El modelo CIPP concibe la evaluación como «el proceso sistemático de identificar, obtener y proporcionar información útil y descriptiva acerca del valor y mérito de las metas, la planificación, la realización y el impacto de lo que se evalúa, con el fin de servir de guía para la toma de decisiones, atender problemas de responsabilidad y promover la comprensión de los fenómenos implicados» (Stufflebeam et al., 1971).

CIPP es el acrónimo de la evaluación de contexto, insumos, procesos y productos. Las evaluaciones del contexto en el que se desarrolla el programa, valora sus fortalezas y debilidades y propone los cambios que pueden realizarse para lograr mejores resultados. Las evaluaciones de entrada evalúan los diferentes modos de proporcionar los servicios que pretende ofrecer el programa, atendiendo a las necesidades de las personas, asegu- 
rando los recursos financieros para conseguir las metas pretendidas. Las evaluaciones de proceso, valoran cómo está funcionando el programa, para proporcionar apoyo y formación al personal, para llevar a cabo las actividades planeadas necesarias. Las evaluaciones del producto, o de resultados, valoran e informan de los resultados conseguidos por el programa.

En general, estas cuatro partes de la evaluación de programas deben responder a las preguntas: ¿Qué se necesita hacer?, ¿Cómo debe ser hecho?, ¿Cómo está siendo realizado? y ¿Tuvo éxito en términos de efectividad, impacto, sostenibilidad y transferencia? (Stufflebeam, 2002, en Martínez Mediano, 2007, p. 154).

El modelo CIPP se guía por principios éticos y profesionales, tal como quedan establecidos en las Normas del Joint Committee on Standards for Educational Evaluation (1994). Este modelo, además, potencia la comunicación de los resultados, el feedback, para todos los afectados o implicados en el programa durante los procesos de evaluación, contribuyendo a su empoderamiento, lo que lleva a un incremento de la motivación y, a su vez, a la mejora del compromiso y de los resultados.

Tanto la garantía de la calidad como la evaluación de programas son elementos esenciales para la mejora continua de los programas y de las organizaciones. Si bien el término «garantía de la calidad» tiene su origen en el ámbito empresarial, dentro del movimiento de la gestión de la calidad orientado a la satisfacción de las expectativas del cliente, este ha sido aceptado en los ámbitos de las organizaciones educativas. De ese modo, algunas universidades han utilizado para la evaluación de su calidad modelos sistémicos procedentes del ámbito empresarial, adaptados a la educación, tales como el Modelo de Excelencia Europeo de la EFQM que se sirve de la autoevaluación del centro para establecer planes de mejora (Martínez-Mediano y Riopérez, 2005), o el de las Normas ISO para la gestión de la calidad que incorporan estrategias para la mejora continua propias de las filosofías de la Calidad Total.

\section{EL PROGRAMA AUDIT}

El programa AUDIT desarrollado por la ANECA (Agencia Nacional de Evaluación de la Calidad y Acreditación), en colaboración con las agencias autonómicas AQU (Agència per a la Qualitat del Sistema Universitari de Catalunya), ACSUG (Axencia para a Calidade do Sistema Universitario de Galicia) y UNIBASQ (Euskal Unibertsitate Sistemaren Kalitate Agentzia), tiene como finalidad orientar a los centros universitarios y universidades en el diseño y la implantación de un Sistema de Garantía Interna de la Calidad (SGIC), sistema que una vez implantado, es evaluado, mejorado y certificado. 
La autonomía que para su gestión tienen las universidades, junto con la transparencia exigida en el marco del EEES, lleva a la responsabilidad de garantizar sus actuaciones y resultados mediante el establecimiento de un SGIC, formalmente establecido y públicamente disponible (AUDIT, 2007).

La garantía de la calidad en el marco del EEES, emana de las recomendaciones elaborados por la ENQA (2005), en colaboración con las agencias de calidad nacionales firmantes del proceso de Bolonia. Dentro de la pluralidad de estrategias existentes para garantizar la calidad de la educación superior se observa una tendencia hacia fórmulas que incorporan la garantía interna de calidad, cimentadas en la responsabilidad de las universidades, junto a procesos de garantía externa de calidad que son responsabilidad de las agencias de evaluación (AUDIT, 2007).

Dentro del marco EEES tanto la evaluación interna, o garantía interna, de las universidades, como la garantía externa de la calidad, a través de las agencias nacionales, para obtener la acreditación de las titulaciones, serán objeto de seguimiento, para garantizar la calidad de la educación y de los criterios de las agencias, en el proceso de la construcción de la convergencia europea en educación superior.

Los criterios y procesos que las agencias de garantía externa de calidad utilizan incluyen una auto-evaluación realizada por la propia universidad; una evaluación externa realizada por un grupo de expertos de acuerdo con los criterios de la agencia; la publicación de un informe incluyendo las decisiones, recomendaciones $\mathrm{u}$ otros resultados formales; $\mathrm{y}$ un procedimiento de seguimiento para revisar las acciones llevadas a cabo por el sujeto del proceso de garantía de calidad a la luz de las recomendaciones contenidas en el informe (ENQA, 2005).

Las auto-evaluaciones realizadas anualmente en las universidades para cada una de sus titulaciones, culminan en un informe de resultados que enfatiza las estrategias institucionales y las actividades para la gestión de la calidad, de las que se informa a la comunidad universitaria. Este proceso de información se constituye en un proceso colectivo institucional de reflexión y de oportunidades para la mejora de la calidad.

Esto es una realidad en el día de hoy en las universidades españolas. Anualmente se realizan informes basados en evaluaciones de proceso, y se comparten en los órganos de representación de la comunidad universitaria como son las Juntas de Facultad. A un nivel superior se informa en los claustros de la Universidad. 
Estos informes, como elementos de evaluación interna y externa, incluyen la memoria de actividades, análisis de indicadores de rendimiento académico, así como indicadores de las fortalezas y estrategias de mejora, análisis de cuestiones de satisfacción de todos los grupos de interés, análisis de aportaciones y valoraciones de equipos docentes sobre el desarrollo de la actividad docente, información y valoración de quejas, plan de mejora a desplegar y seguimiento de las recomendaciones realizadas por ANECA sobre el informe del período anterior.

Estos informes son comunicados a la ANECA que en sus funciones de evaluación o aseguramiento de la calidad externa, emite valoraciones, recomendaciones de mejora y acciones para su seguimiento.

\section{Documentos del Programa AUDIT}

AUDIT orienta el diseño y el despliegue de un SGIC para su certificación. Estas orientaciones vienen recogidas en una serie de documentos que a continuación se describen brevemente:

- Documento 1. Guía para el diseño de Sistemas de Garantía Interna de Calidad de la formación universitaria (Versión 1.0-21/06/07). Recoge los fundamentos para el diseño del SGIC en los centros universitarios, describe el contexto por el que surge la necesidad de crear un SGIC y las bases en las que se fundamenta el diseño del mismo. Por otro lado recoge un plan de todas las fases de aplicación del programa AUDIT, centrándose en las tres primeras fases de diseño del SGIC («Compromiso», «Planificación», «Diagnóstico»), previas a la «Definición y Documentación de los SGIC» (recogida en los documentos 2 y 3 ).

- Documento 2. Directrices, definición y documentación de Sistemas de Garantía Interna de Calidad de la formación universitaria. (Versión 1.0-21/06/07). Es la continuación del anterior, centrándose en la «Definición y Documentación de los SGIC», cuarta fase dentro del diseño de un SGIC. El documento presenta un conjunto de directrices, a modo de ejemplos, para el diseño del SGIC; estas, junto con el documento 3, facilitarán la fase 3 de diagnóstico y, a partir de este, se tiene la información necesaria para realizar la definición y documentación del SGIC. El documento 2 recoge la estructura de cada uno de estos aspectos para definir la documentación y un glosario de términos. 
- Documento 3. Herramientas para el Diagnóstico en la implantación de Sistemas de Garantía Interna de Calidad de la formación universitaria. (Versión 1.0-06/07/07). Recoge diagramas de flujo, a modo de ejemplos, con algunas de las principales actividades que podrían desarrollarse para dar respuesta a cada una de las directrices recogidas en el documento 2. Describe también un conjunto de cuestionarios que pueden servir para evidenciar si se han superado, y en qué grado, las principales etapas que se recogen en los diagramas. Recoge, por último, unas listas de chequeo que permiten la verificación de las evidencias, principalmente documentos o registros, en las que se apoyan las respuestas de los cuestionarios.

- Documento 04. Guía de Evaluación del diseño del Sistema de Garantía Interna de Calidad de la formación universitaria. (Versión 02_13/02/08). Recoge la fase de verificación o evaluación del diseño del SGIC, sus etapas, los requisitos mínimos de la documentación elaborada por el centro, los criterios generales de la evaluación, el protocolo de evaluación, el informe de evaluación y su publicación, y concluye con los criterios referidos a las comisiones de evaluación.

- Documento 05. Guía del Modelo de Certificación de la implantación de Sistemas de Garantía Interna de Calidad. Documento 05. Versión ANECA (Noviembre 2013). Describe las características y las fases de la certificación de los SGIC implantados según el programa AUDIT. Además de las fases del modelo de certificación, el documento describe el camino a recorrer por un centro universitario en la certificación voluntaria de su SGIC, a través de auditorías internas y externas realizadas por auditores especializados. La certificación muestra la correcta implantación y eficacia del SGIC según el modelo del programa AUDIT.

\section{LA TQM Y LAS NORMAS ISO}

Los principios fundamentales de la Gestión de la Calidad Total, en inglés Total Quality Management (TQM) son la búsqueda de la satisfacción de los clientes, el liderazgo de la dirección, la cooperación interna y el trabajo en equipo, la cooperación con clientes y proveedores, la implicación y compromiso de todos los trabajadores, la formación y aprendizaje continuos, la mejora continua y el enfoque en las personas.

Pero estos principios no han sido siempre los mismos. El concepto de la gestión de la calidad ha ido evolucionando desde el control de la calidad, 
entendido como inspección separada del diseño y del proceso de producción, hasta la gestión de la calidad total que se conoce hoy.

Brevemente, se introduce su evolución. El ingeniero Shewart, en la década de los 20, del pasado siglo, desarrolló el control estadístico de procesos, conocido como control estadístico de la calidad, y desarrolla el ciclo de mejora continua: plan, do, study and act. Deming, colaborador de Shewart, sería su principal difusor fuera de los Estados Unidos, conociéndose como el círculo de Deming o de la mejora continua.

En 1951, como estrategia del grupo de los aliados que habían ganado la II Guerra Mundial, enviaron a Deming a Japón, para contribuir al desarrollo de su industria, a impartir un ciclo de conferencia a la Union of Japanese Scientists and Engineers. Introdujo el concepto de calidad, los métodos estadísticos para el control de la calidad y un nuevo concepto de filosofía de gestión empresarial, incluido el ciclo de mejora continua PDCA (plan, do, check, action).

Pero la mayor influencia de Deming en Japón, siguiendo a Tsutsui (1996), no fueron sus análisis estadísticos, sino la defensa de los métodos participativos en la gestión del trabajo, los grupos de trabajo, y la cooperación. Para Deming, la gestión de la calidad solo podía ser lograda con la cooperación de todos los interesados en la mejora continua de la organización.

Juran también fue invitado a Japón en 1954 por la Union of Japanese Scientists and Engineers. Para él, el control de la calidad tenía que ir más allá del control estadístico y los resultados, abarcando todo e implicando a todos los empleados. Desarrolló estrategias de implementación, contemplando aspectos organizativos como los consejos de calidad y los equipos de calidad. Desarrolló la trilogía de la calidad que atendía a los procesos básicos de la misma: la planificación, el control y la mejora. El objetivo de la trilogía era desarrollar productos y procesos que cumplieran con los requerimientos de los clientes.

Con estas ideas Juran dio nuevos desarrollos a la calidad en Japón, aportando un nuevo enfoque más dinámico sobre la TQM de la que ya había hablado Feigenbaum (1961).

Dentro del movimiento de la TQM surgen los sistemas de gestión de la calidad (SGC) que ponen en práctica los principios de este movimiento. Los SGC son modelos para la gestión, herramientas que sirven a las organizaciones de referente y guía en los procesos permanentes de eficacia, satisfacción de los clientes y de mejora continua. Guían a la organización ofreciéndoles las especificaciones sobre qué tipo de requisitos deben de implementar para poder brindar productos y/o servicios de alto nivel. 
Desde los 90, uno de los SGC más utilizados en la empresa es el basado en la Norma UNE-EN ISO 9001, que es utilizado también en educación y que puede servir, bien desarrollado, como un medio relevante y eficaz al servicio de proyectos educativos de calidad (Pérez Juste, 2005). Estas normas y sus SGC se basan en los ocho principios de gestión de la calidad siguientes:

1. ${ }^{\circ}$ Enfoque al cliente: Las organizaciones dependen de sus clientes y por lo tanto deberían comprender las necesidades actuales y futuras de los clientes, satisfacer los requisitos de los clientes y esforzarse en exceder las expectativas de los clientes.

2. ${ }^{\circ}$ Liderazgo: Los líderes establecen la unidad de propósito y la orientación de la organización. Ellos deberían crear y mantener un ambiente interno, en el cual el personal pueda llegar a involucrarse totalmente en el logro de los objetivos de la organización.

3. ${ }^{\circ}$ Participación del personal: El personal, a todos los niveles, es la esencia de una organización, y su total compromiso posibilita que sus habilidades sean usadas para el beneficio de la organización.

4. ${ }^{\circ}$ Enfoque basado en procesos: Un resultado deseado se alcanza más eficientemente cuando las actividades y los recursos relacionados se gestionan como un proceso.

5. Enfoque de sistema para la gestión: Identificar, entender y gestionar los procesos interrelacionados como un sistema, contribuye a la eficacia y eficiencia de una organización en el logro de sus objetivos.

6. ${ }^{\circ}$ Mejora continua: La mejora continua del desempeño global de la organización debería ser un objetivo permanente de esta.

7. Enfoque basado en hechos para la toma de decisión: Las decisiones eficaces se basan en el análisis de los datos y la información.

8. ${ }^{\circ}$ Relaciones mutuamente beneficiosas con el proveedor: Una organización y sus proveedores son interdependientes, y una relación mutuamente beneficiosa aumenta la capacidad de ambos para crear valor.

El SGC de acuerdo con ISO 9001 en el ámbito de una organización educativa, debe entenderse en relación con los procesos de diseño del producto como planes de estudio y diseños curriculares, su desarrollo mediante los procesos de enseñanza y aprendizaje, los procedimientos de implementación incluidos la estructura organizativa y los recursos o la planificación docente, y la medición de los resultados. 
El control del producto educativo, del resultado que se pretende, se realiza desde el diseño de los programas educativos y su viabilidad, su planificación, su realización y la evaluación de sus resultados, en todos los grupos de interés y en todos los servicios específicos.

El SGC debe contemplar, entre otros requisitos, las responsabilidades de la dirección y su compromiso con la calidad y su diseño, aplicación y evaluación; la gestión de los recursos, incluidos infraestructuras, formación y clima de trabajo; la realización del producto, incluida la planificación, la revisión en contraste con el diseño, la realización y los resultados; la medición, análisis y mejora continua, mediante auditorías internas, revisiones de la dirección, valoraciones de la satisfacción del cliente, no conformidades y otras fuentes.

\section{EL PROGRAMA AUDIT Y LAS NORMAS ISO. ANÁLISIS COMPARATIVO}

Para nosotros, el programa AUDIT y las normas ISO son modelos de calidad que surgen en sectores productivos distintos pero que comparten algunas de sus estrategias y fines. A continuación vamos a hacer una comparación de los mismos, de sus objetivos y principios, a nivel teórico, y de su puesta en práctica, centrándonos en sus fases y documentos.

\section{Objetivos}

«El programa AUDIT persigue dos objetivos básicos: a. Facilitar a los Centros orientaciones para el diseño de Sistemas de Garantía Interna de Calidad de las enseñanzas que imparten. b. Poner en práctica un procedimiento que conduzca al reconocimiento del diseño de dichos sistemas, por parte de las agencias de garantía externa de la calidad» (ANECA 2007, Documento 1, p. 14).

Por otro lado, «la familia de Normas ISO 9000 [...] se ha elaborado para asistir a las organizaciones, de todo tipo y tamaño, en la implementación y la operación de sistemas de gestión de la calidad eficaces [...]. La Norma ISO 9001 especifica los requisitos para los sistemas de gestión de la calidad aplicables a toda organización que necesite demostrar su capacidad para proporcionar productos que cumplan los requisitos de sus clientes y los reglamentarios que le sean de aplicación, y su objetivo es aumentar la satisfacción del cliente [...]» (ISO 9000:2005, introducción, VI).

Las normas ISO definen unos requisitos mínimos para el diseño de un sistema de gestión de la calidad (equivalente al objetivo a de AUDIT), que 
pueden ser reconocidos por entidades certificadoras acreditadas (equivalente al objetivo b de AUDIT).

\section{Principios fundamentales}

Los ocho principios para la gestión de calidad de las normas ISO se localizan también en AUDIT:

El desarrollo de un SGIC según AUDIT debe contemplar «la planificación de la oferta formativa, la evaluación y revisión de su desarrollo, así como la toma de decisiones para la mejora de la formación» (ANECA 2007, Documento 1, p. 13). A través del mismo la universidad podrá analizar sus resultados, diseñar mejor su oferta formativa, revisar el desarrollo de la misma e introducir los cambios necesarios en un ciclo de mejora continua. Esto no es más que el utilizar la información basándose en hechos reales para mejorar, a partir de la satisfacción y las necesidades de sus clientes internos y externos, de las evidencias o de las mejoras dadas en su diseño (principios 1, 3, 6, 7 de gestión de la calidad).

Para AUDIT, la gestión de los procesos y de estos como sistema (principios 4 y 5 de gestión de la calidad) se revela importante al aconsejar, dando ejemplos en el Documento 3 (ANECA, 2007), la descripción de los procesos en diagramas de flujo como forma de ordenar de forma coherente las actividades implicadas de un proceso.

Las universidades deben responder a las expectativas sociales y a la confianza que la sociedad ha depositado en ellas y en su autonomía. Sus líderes (principio 2 de gestión de la calidad) deben responder a lo que la sociedad les demanda al depositar su confianza en la organización, de ahí que se hagan necesarias una buena gestión, una mejora continua y una satisfacción de las demandas, aspectos todos relacionados con la calidad universitaria.

Además, los SGIC, según AUDIT, sistematizan la mejora continua necesaria dentro de la organización (principio 6 de gestión de la calidad). A través de sus actuaciones internas (responsabilidad de las universidades) y externas (responsabilidad de las agencias de evaluación y calidad) los centros universitarios establecen un SGIC que opta por el control y la mejora continua del mismo y de su servicio, en este caso, la oferta formativa. El mismo ciclo de mejora continua de la Formación Universitaria (ANECA, 2007, Documento 1, p. 13) recuerda al ciclo de Deming contextualizado en la educación universitaria. Y el propio desarrollo de AUDIT incorpora esta mejora cuando tras la verificación del diseño de un SGIC, se añade una fase de mejora del mismo antes de su implantación. 
Por último, para AUDIT los grupos de interés y la participación de todas las personas son fundamentales para el funcionamiento de la organización (principios 3 y 8 de gestión de la calidad). El compromiso del personal de la universidad, tanto interno como externo, incluyéndose los proveedores considerados como las personas u organizaciones que prestan productos o servicios a la universidad, debe ser utilizado para el beneficio y el crecimiento de la organización.

\section{Criterios de garantía de calidad del Programa AUDIT y Requisitos de la Norma ISO 9001}

El programa AUDIT describe unos criterios de garantía de calidad (ANECA, 2007, Documento 1) elaborados a partir de las Directrices para la elaboración de títulos universitarios de grado y máster (MEC, 2006) y de los Criterios y directrices para la garantía de calidad en el Espacio Europeo de Educación Superior (ENQA, 2005).

Los SGIC basados en AUDIT deben recoger estos criterios con, a nuestro entender, una doble finalidad: por un lado normalizan, regulan lo que deben contener, es decir, sirven de «marco de referencia común» (ANECA, 2007, Documento 1, p. 11) para los centros y las agencias tanto en el diseño como en la evaluación del sistema; y por otro lado, aseguran que, teniéndolos recogidos, los procesos particulares de cada centro sean también de calidad.

Exponemos los siete a continuación:

1. Política y objetivos de calidad: Los SGIC deben describir cómo cada centro trabaja en pro de una cultura de la calidad, que a su vez se apoya en una política y objetivos de calidad conocidos por todos.

2. Diseño de la Oferta formativa: Los SGIC deben recoger los mecanismos existentes en los centros de garantía de la calidad de su oferta formativa, así como de su actualización.

3. Desarrollo de la enseñanza y otras actuaciones orientadas a los estudiantes: Este criterio se centra en la existencia de procedimientos para garantizar y promover el aprendizaje del estudiante, procedimientos que debe recoger el SGIC.

4. Personal académico y de apoyo a la docencia: En cada centro deben existir mecanismos de control y mejora de su profesorado, estos mecanismos deben recogerse en el SGIC. 
5. Recursos materiales y servicios: Se refiere a la existencia de mecanismos de gestión y mejora de servicios y recursos para el aprendizaje, al igual que los anteriores deben diseñarse dentro del SGIC.

6. Resultados de la formación: La existencia (y diseño dentro del SGIC) de procedimientos de búsqueda, análisis y utilización de datos de resultados para la mejora.

7. Información pública: Cada centro (y SGIC) debe asegurar la existencia de mecanismos para la publicación periódica de información actualizada relativa a las titulaciones y a los programas.

Estos son, por tanto, unos criterios de evaluación normalizados para AUDIT, que pueden ser comparados con los requisitos de la norma ISO 9001, como vemos en la tabla 1. A nuestro entender, ISO incluye unos requisitos más generales y abiertos a un mayor número de organizaciones de diferentes sectores, siendo los criterios de AUDIT más concretos para la formación universitaria.

Tabla 1

Comparación de los Criterios de AUDIT con los Requisitos de la Norma ISO 9001

CRITERIOS DE AUDIT

1. Política y objetivos de calidad

2. Diseño de la Oferta formativa

3. Desarrollo de la enseñanza y otras actuaciones orientadas a los estudiantes

4. Personal académico y de apoyo a la docencia

5. Recursos materiales y servicios

6. Resultados de la formación
6. Gestión de los recursos

5.4.1. Objetivos de la calidad

7. Realización del producto

REQUISITOS DE LA NORMA ISO 9001

5.3. Política de la calidad

6.2. Recursos humanos

5.6. Revisión por la dirección

8. Medición, análisis y mejora

7.2. Comunicación con el cliente

5.5.3. Comunicación interna

Si analizamos uno a uno los criterios establecidos por AUDIT para diseñar un SGIC y los comparamos con los requisitos de la norma ISO 9001 necesarios para establecer un SGC, podemos ver el parecido existente: 
$\mathrm{Al}$ igual que AUDIT, ISO establece la obligación de describir la política y los objetivos de calidad. La realización del producto (sus procedimientos, recursos, actividades necesarias, etc.) debe incluirse también en ambos, aunque AUDIT concreta más, centrándose en la formación ofertada y en la promoción del aprendizaje. Los recursos humanos para ISO deben identificarse y formarse para la mejora, el personal académico tanto para AUDIT como para ISO, también. Y la mejora es un principio fundamental para ambos, al igual que la medición de los resultados y su análisis para la toma de decisiones.

La gestión de los recursos que apoyan la actividad (la enseñanza y el aprendizaje para AUDIT) son de obligado cumplimiento para ambos: infraestructuras, ambientes de trabajo y servicios de calidad son necesarios para que el proceso y producto sean de calidad. Quizás quepa la comparación en la gestión de las compras de productos o servicios que influyen en la actividad de la organización. Mientras AUDIT recoge esta sistemática en su directriz 1.4. exponiendo que «El Centro/Universidad debe dotarse de mecanismos que le permitan diseñar, gestionar y mejorar sus servicios y recursos materiales para el adecuado desarrollo del aprendizaje de los estudiantes» (ANECA, 2007, Documento 2, p. 9), ISO en su requisito 7. Realización del producto, lo hace más exhaustivo y de obligado cumplimiento. En esta definición más delimitada del proceso de compras, ISO establece que los productos/servicios adquiridos cumplan con lo especificado, instaura un control y/o evaluación a los proveedores, la descripción de los productos a comprar y su verificación una vez comprado.

Por último, AUDIT detalla el criterio Información pública que es comparable al requisito de norma ISO Comunicación con el cliente. AUDIT centra su información al exterior en la publicación periódica relativa a las titulaciones y programas, y a los resultados de estudiantes, lo que podríamos considerar como su producto. ISO, desde su perspectiva, habla también de la comunicación con el cliente en cuanto a la información del producto, las consultas, contratos o atención a pedidos, y la retroalimentación del cliente (información de este sobre el producto, lo que incluye las quejas o reclamaciones sobre el producto).

\section{Fases}

A la hora de diseñar, implantar y certificar un SGC según ISO, desde nuestra experiencia, vemos necesarias las siguientes fases:

1. Decisión de la implantación de un SGC.

2. Definición de objetivos a conseguir y de etapas a seguir. 
3. ${ }^{\circ}$ Formación del profesorado en calidad y sistemas de gestión de la calidad.

4. ${ }^{\circ}$ Creación del departamento de calidad.

5. ${ }^{\circ}$ Diseño del sistema de calidad.

6. ${ }^{\circ}$ Formación en el sistema y difusión entre los trabajadores del mismo.

7. ${ }^{\circ}$ Implantación y rodaje del sistema.

8..$^{\circ}$ Evaluación del sistema y mejora.

9. ${ }^{\circ}$ Solicitud de certificación.

$10 .^{\circ}$ Auditoría interna y plan de mejora.

11. ${ }^{\circ}$ Visita previa del organismo certificador y plan de mejora.

12. ${ }^{\circ}$ Auditoría del organismo certificador y plan de mejora. Posible certificación.

13. ${ }^{\circ}$ Mantenimiento y mejora del sistema.

14. ${ }^{\circ}$ Auditorías de seguimiento y renovación de la certificación

Si comparamos estas fases con las descritas para desarrollar el programa AUDIT (ANECA, 2007, Documento 1, p. 16) podemos ver que los pasos a seguir son prácticamente los mismos.

Quizás la mayor diferencia es que mientras que para AUDIT, la verificación del diseño del SGIC se realiza en la Fase 2, tras su definición y de forma previa a su implantación y participación en su certificación, para ISO, el organismo certificador comprueba que el diseño del SGC es acorde con la norma una vez implantado y realizada la solicitud de certificación, esto es, antes o durante la visita previa a la organización.

\section{Documentos básicos de un SGIC}

Aquella organización que diseñe un SGC según ISO 9001, debe elaborar de forma obligatoria una política y unos objetivos de calidad, un manual de calidad que recoja la descripción de todos los requisitos de la norma, unos documentos de la organización como el mapa de procesos, fichas de procesos, procedimientos, etc., y unos procedimientos documentados obligatorios. Estos procedimientos están relacionados con el control de los 
documentos y de los registros, con la auditoría interna, con las no conformidades y con las acciones correctivas y/o preventivas.

El programa AUDIT parece ser más flexible en torno a la documentación obligatoria del SGIC. Deja abierto a cada centro universitario su definición de la documentación del sistema, teniendo en cuenta que esta debe recoger los criterios antes descritos y otros aspectos como los objetivos y principios de actuación, los grupos de interés, el alcance, las fases, los procedimientos, y la organización, estructura y recursos.

En este sentido el documento 2 (ANECA 2007, p. 4) recoge que «se considera una buena práctica documentar todos los aspectos relativos a los Sistemas de Garantía Interna de Calidad en forma de procedimientos, normas, manuales, fichas de proceso, diagramas, etc.».

Además exige unos requisitos básicos para la documentación (ANECA 2008, Documento 4, pp. 11 y 13) que recuerdan fácilmente a la gestión documental y los requisitos de la documentación descritos por ISO.

Por último, si damos un repaso a la documentación de algunas universidades que han elaborado su SGIC podemos ver que la mayoría han definido un manual de calidad, como documento marco donde se recogen los criterios y aspectos antes descritos, y por otro lado, describen los procesos en flujogramas que se llevan a cabo para la formación universitaria. Estos procesos recuerdan a los operativos, estratégicos y de soporte que se definen también con ISO.

\section{Certificación de la Implantación y Auditorías}

El modelo de certificación de la implantación de un SGIC según el programa AUDIT se desarrolla en el documento 5 (ANECA, 2013). Haciendo un análisis del mismo podemos comprobar el parecido que tiene con ISO en lo relativo a las fases de auditoría interna/externa y certificación.

Para ISO 9000, las auditorías se utilizan para determinar el grado en que se han alcanzado los requisitos del SGC, en otras palabras, determinan si el SGC está implantado conforme a los requisitos establecidos y a la norma en la que se basa. Sus hallazgos se utilizan para evaluar la eficacia del SGC y para identificar oportunidades de mejora. La certificación puede considerarse como un registro de conformidad con los requisitos contenidos en una norma como la ISO 9001. Estos objetivos son idénticos en AUDIT, donde la fase de la certificación de los SGIC sirven para comprobar su implantación y su nivel de eficacia a través de la realización de auditorías in- 
ternas o evaluación (recomendado), de revisiones de la documentación, de auditorías externas, de Planes de Acciones de Mejora, y de auditorías internas, externas o autoinformes de seguimiento del certificado (ANECA, 2013).

La única diferencia es que AUDIT puntualiza una metaevaluación para una posible mejora del proceso de certificación.

Por último, ISO describe como requisito obligatorio un procedimiento documentado que detalle la sistemática de las auditorías internas en la organización. AUDIT no lo hace obligatorio pero sí aconsejable.

Otro aspecto característico de AUDIT es su alineación con otros programas desarrollados por las agencias de calidad para evaluar las titulaciones oficiales como son VERIFICA, MONITOR y ACREDITA centrados, respectivamente, en la evaluación del diseño de los planes de estudio, en el seguimiento de la implantación de los mismos y en su acreditación.

Con la intención de ahorrar esfuerzos y recursos, en las universidades con un SGIC auditado y certificado por AUDIT, determinados aspectos de la evaluación de sus títulos quedan eximidos de volver a evidenciarse, simultaneando de esta forma la evaluación del SGIC con la de los títulos.

\section{CONCLUSIONES}

El programa AUDIT ha supuesto una revolución en el campo de la calidad universitaria española. Por un lado, siendo el primer aspecto a destacar, normaliza, sistematiza y suma una serie de prácticas de la garantía de la calidad que se estaban dando a nivel nacional. Esta normalización se da en unos documentos que aportan unas directrices comunes basadas en el trabajo de la ENQA dentro del marco común del EEES. Estos documentos permiten la planificación, la evaluación y la toma de decisiones para la mejora de la calidad de la oferta formativa.

Por otro lado, desarrolla en el contexto nacional universitario los principios y prácticas de la gestión de la calidad total que se vienen dando a nivel internacional y en todo tipo de organizaciones.

Otro aspecto positivo del programa es que establece, definitivamente, una evaluación institucional de la universidad española en coherencia con el resto de países europeos. Con esta metodología evaluativa la universidad puede responder, desde su autonomía universitaria, a las exigencias de mejora de su calidad, y proporciona a la sociedad información que puede usarse para la toma de decisiones. 
Dentro de las evaluaciones que se hacen del diseño de los SGIC según AUDIT, los evaluadores cuentan con unos criterios de evaluación: el documento 4 (ANECA, 2008). Estos criterios de evaluación no existen en ISO. A la hora de implantar un SGC según ISO, la organización elabora una documentación que debe cumplir con los requisitos de dicha norma. El diseño de esta documentación no es evaluada por parte de los organismos certificadores hasta antes de las auditorías de certificación. Las organizaciones que desean certificar su SGC no tienen herramientas o instrumentos para evaluar el diseño de su SGC salvo lo recogido por la propia norma ISO 9001.

Este documento 4 de AUDIT (ANECA, 2008) es un instrumento útil al igual que el anexo VII del documento 5 (ANECA, 2013) «Protocolo de auditoría de implantación del SGIC» que puede ser utilizado por los auditores para comprobar si las directrices de AUDIT recogidas en el documento 2 (ANECA, 2007) se llevan a cabo en el centro auditado.

Además, dentro de esta filosofía, destacan algunos principios de los cuales nos parece especialmente relevante el de la mejora continua. Un principio que aparece tanto en la disciplina de la evaluación de programas como en el movimiento de la TQM que hemos analizado. Evaluar y mejorar son dos conceptos inseparables en los procesos de calidad. Por un lado, la evaluación de programas se realiza para mejorarlos y para conseguirlo necesita información proveniente del proceso de evaluación. Por otro lado, la evaluación y la mejora son elementos intrínsecos en el ciclo Deming o PDCA. AUDIT sirve de medio para que los centros establezcan una rutina, una dinámica para mejorar continuamente todos los ámbitos deseados. Otro punto fuerte del programa.

Por último, como aspecto positivo destacable, la elaboración de un SGIC que debe ser supervisado de forma externa por las agencias (creando una evaluación desde tres puntos diferentes: garantía interna, garantía externa y acreditación), generan un punto de encuentro entre estas y las universidades. Acercamiento necesario para el desarrollo de la evaluación, la calidad y la mejora de ambas instituciones.

Con AUDIT se crea una documentación y una definición de los procesos que se llevan a cabo para garantizar que trabajamos en pro de la calidad y la satisfacción de las necesidades de los grupos de interés a través de la propia evaluación de la universidad. Un modelo de evaluación que recoge las ideas de la evaluación de programas y del movimiento de la TQM, corrientes muy distintas que aportan muchas ventajas a quienes las utilizan.

La certificación del modelo de calidad por ISO o AUDIT se hace secundaria, lo importante es la definición, implantación y mantenimiento de 
un SGIC efectivo. Trabajar a través del mismo, puede mejorar la eficacia, eficiencia y pertinencia de la universidad.

Creemos manifiestamente que AUDIT se hace imprescindible al personalizar los principios de la calidad total y de la evaluación institucional en un SGIC adaptado al contexto educativo universitario nacional y al formar parte de un nuevo marco europeo en pro de un EEES de calidad en el que han quedado homologados planes de estudio, créditos, movilidad y, por último, garantía de calidad. 


\section{REFERENCIAS BIBLIOGRÁFICAS}

Agencia Nacional de Evaluación de la Calidad y Acreditación - -ANECA (2007). Programa AUDIT. Herramientas para el diseño. Documentos 1, 2, y 3. Recuperado de: http://www.aneca.es/ Programas/AUDIT/Herramientas-parael-diseno

Agencia Nacional de Evaluación de la Calidad y Acreditación - -ANECA (2008). Programa AUDIT. Herramientas para la evaluación. Documento 4. Recuperado de: http://www.aneca. es/Programas/AUDIT/Herramientaspara-la-evaluacion

Agencia Nacional de Evaluación de la Calidad y Acreditación - ANECA (2013). Programa AUDIT. Documento 5. Versión ANECA. Recuperado de: http://www.aneca.es/Programas/ AUDIT/Fase-de-certificacion-de-la-implantacion-de-los-SGIC

Alkin, M. C. (1969). Evaluation theory development. Evaluation Comment, 2, 1.

Declaración de Bolonia (1999, Junio). Declaración conjunta de los Ministros Europeos de Educación. Bolonia, Italia. Recuperado de: www.educacion.es/dctm/boloniaeees/ documentos/02que/declaracion-bolonia. pdf?documentId=0901e72b8004aa6a

European Association for Quality Assurance in Higher Education - ENQA (2005). Criterios y Directrices para la Garantía de Calidad en el Espacio Europeo de Educación Superior. Documento aprobado por los ministros europeos de educación. Bergen, Noruega.

Feigenbaum, A. V. (1961). Total Quality Control: Engineering and Management. New York: McGraw-Hill.

ISO 9000:2005. Sistemas de gestión de la calidad. Fundamentos y vocabulario. Ginebra: International Standard Organization.
ISO 9001:2008. Sistemas de gestión de la calidad. Requisitos. Ginebra: International Standard Organization.

Martínez Mediano, C. (2007). Evaluación de programas educativos. Modelos $y$ procedimientos. Madrid: UNED.

Martínez Mediano, C., y Riopérez Losada, N. (2005). El Modelo de Excelencia de la EFQM y su aplicación para la mejora de la calidad de los Centros Educativos. Educación XX1, 8, 35-65.

MEC (2006). Directrices para la elaboración de títulos universitarios de Grado y Máster. Documento de trabajo. Madrid: Ministerio de Educación y Ciencia.

Pérez Juste, R. (2005). Calidad de la educación. Calidad en educación. Hacia la necesaria integración, Educación XX1, 8, 11-33.

Stufflebeam, D. L. (2002). CIPP Evaluation Model checklist. Evaluation checklists project. Recuperado de: www.wmich.edu.

Stufflebeam, D. L. et al. (1971). Educational evaluation and decisión making. Itasca, ILL: Peacock.

Stufflebeam, D. L., y Shinkfield, A. J. (1987). Evaluación sistemática. Guía teórica y práctica. Barcelona, Buenos Aires: Paidós- MEC.

Stufflebeam, D. L., y Shinkfield, A. J. (2007). CIPP Model for evaluation. An improvement/accountability approach. En D. Stufllebeam (Ed.) Evaluation Theory, models, and applications (pp.325365). San Francisco: Jossey-Bass.

The Joint Committee On Standards For Educational Evaluation (1994). The Program Evaluation Standards. Thousand Oaks, CA: Sage P. Inc.

Tsutsui, W. M. (1996). W. Edwards Deming and the Origins of Quality Control in Japan. Journal of Japanese Studies, 22(2), 295-325. 


\section{PERFIL ACADÉMICO Y PROFESIONAL DE LOS AUTORES}

Jorge Antonio Arribas Díaz, Maestro por la Universidad de Jaén y licenciado en Antropología social y cultural por la Universidad de Sevilla, en la actualidad realiza su tesis doctoral centrada en los Sistemas de gestión de la calidad según la norma ISO 9001 en el departamento de Métodos de Investigación y Diagnóstico en Educación de la UNED en Madrid. Ha sido gestor de la calidad, auditor interno y formador sobre calidad, además de maestro, en la Fundación SAFA en Andalucía.

Catalina Martínez Mediano, Doctora por la Universidad Nacional de Educación a Distancia, con premio extraordinario. Profesora Titular U. UNED. Sus intereses son el Aprendizaje, la Investigación Evaluativa y la Calidad. Investigadora principal en proyectos competitivos (UE) InternetBased Performance Support Systems with Educational Elements (IPSS EE); Distributed Internet-Based Performance Support Environment for Individualized Learning (DIPSEIL); On-Line Learning Mathematics and Science; Internet-Based Performance-centered Learning Environment for Curriculum Support (IPLECS). En 2011 disfrutó de la beca Salvador de Madariaga del MEC España.

Dirección de los autores: UNED

Facultad de Educación

Dpto. MIDE I

C/. Juan del Rosal, 14

28040 Madrid

E-mail: jorgearribasdiaz@gmail.com

cmarme@edu.uned.es

Fecha Recepción del Artículo: 19. Enero. 2013

Fecha Modificación del Artículo: 01. Julio. 2013

Fecha Aceptación del Artículo: 16. Julio. 2013

Fecha Revisión para la publicación: 18. Enero. 2015 
